\title{
PAPER
}

\section{A practical definition of conduction block in Ivlg responsive multifocal motor neuropathy}

\author{
A Ghosh, M Busby, R Kennett, K Mills, M Donaghy
}

J Neurol Neurosurg Psychiatry 2005;76:1264-1268. doi: 10.1136/jnnp.2004.047688

\begin{abstract}
See end of article for authors' affiliations

.....................

Correspondence to:

Dr M J Donaghy,

University Department of

Clinical Neurology,

Radcliffe Infirmary, Oxford

OX2 6HE, UK;

joanna.wilkinson@

clneuro.ox.ac.uk
\end{abstract}

Received 16 June 2004

Revised version received

25 November 2004

Accepted 1 December 2004
Background: Multifocal motor neuropathy with conduction block (MMN) can be mistaken for motor neurone disease or other lower motor neurone syndromes, but is treatable with intravenous immunoglobulin (lvlg). Formal electrophysiological criteria for conduction block (CB) are so stringent that substantial numbers of patients may miss out on appropriate treatment.

Methods: Electrophysiological data were collected from 10 healthy volunteers and compared to data from 10 patients who satisfied the clinical criteria for $M M N$ and who responded to Ivlg. This produced a definition of $C B$ in MMN patients which was compared with existing definitions to assess "miss rates". Results: Mean values for compound muscle action potential area, amplitude, and duration were calculated in normal subjects. Results beyond 3 SD of their respective means were considered abnormal. Using these criteria, $C B$ in the context of $M M N$ was defined as a reduction in negative peak area $>23 \%$ along a distal nerve segment or $>29 \%$ across a proximal segment; or a reduction in amplitude $>32 \%$ across a distal segment or $>33 \%$ across a proximal segment. All Ivlg responsive patients had at least one nerve segment showing such CB. Employing some criteria from the literature would have denied treatment to over $30 \%$ of responsive patients.

Conclusion: In the clinical setting of suspected $M M N$, less stringent criteria for $C B$ can improve the diagnosis of this treatable disorder. Exclusions on grounds of temporal dispersion may be over-restrictive. A little over one third of $\mathrm{CBs}$ occur proximally.
M ultifocal motor neuropathy (MMN) is an uncommon disorder characterised by slowly progressive asymmetrical lower motor neurone weakness usually starting in the arms with minimal or no sensory symptoms. ${ }^{1-3}$ Relative lack of wasting even in severely weakened muscles is a distinctive early clinical feature. ${ }^{4} \mathrm{MMN}$ can be successfully treated with intravenous immunoglobulin (IvIg)..$^{5-11}$ It is crucial to distinguish this treatable condition from other motor neurone diseases.

The electrophysiological hallmark of MMN is conduction block (CB), in which an action potential fails to propagate through a segment of an intact axon. Various working definitions of CB have been proposed..$^{10-21}$ The presence of any temporal dispersion (TD) has been seen as a major obstacle to satisfactory definition of CB. Many of the existing definitions of $\mathrm{CB}$ either do not accommodate TD or demand even more stringent criteria in its presence. This could reduce sensitivity for electrophysiological detection of this potentially treatable disorder. Tighter criteria for defining CB demand more protracted neurophysiological assessment, which may be impractical in many healthcare settings. We have explored less stringent definitions of CB in patients with MMN, with a view to increasing sensitivity for identifying IvIg responsive patients.

\section{METHODS}

Sensory and motor neurophysiological examinations were performed in 10 healthy controls using standardised techniques. The mean age in this group was 40.3 (range 3158) years. Distal sensory responses were assessed in the median and ulnar nerves, measuring peak-to-peak amplitudes and conduction velocities. Motor stimulation of peripheral nerves at the wrist, elbow, axilla, and Erb's point was performed at standard sites with a Devices Type 3072 stimulator (Digitimer, Welwyn Garden City, Herts, UK) and a conventional bipolar stimulating electrode. Stimulation of the C8 root was performed using a Devices Type 180 stimulator (Digitimer) and saline soaked lint covered with silver electrodes (each electrode area $\mathrm{l} \mathrm{cm}^{2}$ ) with the cathode positioned in the space between the spinous processes of the C7 and Tl vertebrae. ${ }^{22}$ Compound muscle action potentials (CMAPs) were recorded using standard $\mathrm{AgCl}$ electrodes and a Medelec Mystro instrument (Medelec, Old Woking, Surrey, UK). For the median nerve, CMAPs were recorded from the left and right abductor pollicis brevis following supramaximal stimulation of the nerve at the wrist, elbow, axilla (in one patient), Erb's point, or over the C8 root. For the ulnar nerve, CMAPs were recorded from the left and right abductor digiti minimi following supramaximal stimulation at the wrist, below and above the elbow, the axilla (in one patient), Erb's point, or over the C8 root. Entrapment sites were excluded from assessment of CB. Measurements were made for negative peak amplitudes, negative peak areas, negative peak durations, distal latencies (DL), conduction velocities, and F-waves. For the last three parameters, the American Academy AIDS Task Force criteria were used to calculate prolongations in the "demyelinating range" ${ }^{\prime 23}$ The percentage reduction in CMAP area and amplitude between two adjacent points of stimulation were calculated using the formula: (dCMAP-pCMAP/dCMAP) $\times 100 \%$, where dCMAP and pCMAP represent distal and proximal CMAP values, respectively. Percentage increase in duration in the proximal segment relative to the distal segment was used to calculate TD using the formula: (pCMAP duration-dCMAP duration/ dCMAP duration) $\times 100 \%$. Nerves with distal CMAP amplitudes of $<1.0 \mathrm{mV}$ were not accepted for measurement of $\mathrm{CB}$ because of the likelihood of errors in measurement.

Abbreviations: $\mathrm{CB}$, conduction block; $\mathrm{CMAP}$, compound muscle action potential; DL, distal latencies; Ivlg, intravenous immunoglobulin; MMN, multifocal motor neuropathy; TD, temporal dispersion 
The mean (standard deviation, SD) percentage drops in CMAP amplitudes and areas and increases in duration across the distal (wrist to elbow) and proximal segments (elbow to neck) for healthy controls were calculated (for explanation, see below). We used values in excess of $3 \mathrm{SD}$ of the respective means to define $\mathrm{CB}$ and TD.

We then applied these values to detect $\mathrm{CB}$ in 10 patients with a clinical diagnosis of MMN who responded to IvIg. All patients had a chronic asymmetric, predominantly distal, lower motor neurone weakness, with disproportionate preservation of muscle bulk and without sensory involvement. A neurologist (MD) with extensive experience in managing peripheral nerve disorders performed the clinical observations while being blinded to the neurophysiological results. Clinical improvement in the IvIg responsive patients was defined as: (i) an improvement in MRC score by at least one grade in one or more affected muscle groups; and (ii) a clear functional improvement in performance of simple activities that had been affected by the patient's weakness. All but one patient remain under follow up at the time of preparing this manuscript, all maintaining their clinical improvement on regular IvIg.

\section{RESULTS}

\section{Controls}

Data were obtained from 37 nerves and 74 nerve segments in healthy controls. Mean (SD) reductions in amplitude and area and mean TD are shown in table 1.

CMAPs from C8 stimulation were either equal or greater in size compared with those recorded from Erb's point. Therefore, for the assessment of area, amplitude, and duration, the proximal segment was defined as the elbow to neck segment. The same was true for eight IvIg responsive patients. In two other IvIg responsive patients, where only limited studies could be performed, the elbow-Erb's point and elbow-axilla segments were taken as the proximal segments, respectively.

\section{MMN patients}

Six of the IvIg responsive patients were males and four were females. The mean (SD) age of onset of symptoms was 44.2 (15.6) years and the mean duration of illness at the start of treatment with IvIg was 10.9 (7.6) years. The upper limb was involved first in six patients, the lower limb in three, and the illness started in both upper and lower limbs in one patient. Finger or wrist drop at or within 1 year of onset was seen in $4 / 10$ patients. Myotonia and/or cramps were present in four patients. Wasting was absent or mild in 5/10 patients, even in severely weakened muscles. Although one patient reported mild sensory symptoms, sensory conduction was normal in all. None of the patients had upper motor neurone signs. Two patients had anti-GMl antibodies. All three patients who had received steroids previously had worsened with that treatment. The average follow up period so far is 48.3

\begin{tabular}{lcc}
\multicolumn{3}{l}{ Table 1 Changes in CMAP values in healthy controls } \\
\hline Nerve segment & Mean (SD) & Mean+3 SD \\
\hline Area reductions & \\
$\quad$ Distal segment & $6.3(5.5) \%$ & $23 \%$ \\
$\quad$ Proximal segment & $7.5(7.1) \%$ & $29 \%$ \\
Amplitude reductions & & \\
$\quad$ Distal segment & $8.5(7.7) \%$ & $32 \%$ \\
$\quad$ Proximal segment & $9.2(8.0) \%$ & $33 \%$ \\
Duration increases & \\
$\quad$ Distal segment & $6.8(9.9) \%$ & $37 \%$ \\
Proximal segment & $9.8(8.7) \%$ & $36 \%$ \\
\hline
\end{tabular}

(25.3) months, ranging from 6 months (in a patient who emigrated shortly afterwards) and 7 years.

Using mean+3 SD values over healthy controls, CB was defined as a reduction in negative peak area $>23 \%$ in a distal segment and $>29 \%$ in a proximal segment, or a reduction in amplitude of $>32 \%$ for a distal segment and $>33 \%$ for a proximal segment (table 1).

Altogether 72 nerve segments were studied in the IvIg responsive group. Each patient in this group had at least one nerve segment with a reduction in area suggestive of $\mathrm{CB}$. Using the area criteria, one patient had one $\mathrm{CB}$, two patients had two CBs, four patients had three CBs, two patients had four CBs, and another patient had five CBs. Twelve of these 30 segments $(40 \%)$ were proximal. Eight patients had area reductions in excess of $50 \%$ in at least one nerve segment. The largest area reductions in the other two patients were $37 \%$ and $40 \%$, both seen in distal segments. Twenty six of the 30 segments where area was reduced also showed a drop in amplitude suggestive of CB. An additional nine segments with amplitude reduction without significant area changes were also present.

TD $>37 \%$ (table 2) was present in 22 nerve segments. These included 15 segments with concomitant reduction both in area and amplitude, three segments with reduction in amplitude only, and the remaining four segments with no evidence of CB.

In the IvIg responsive group, DL were prolonged into the demyelinating range in two nerves in different patients. Conduction velocities lay in the demyelinating range in one nerve in a separate patient and were found within segments with CB. Out of 36 nerves studied, F-latencies were prolonged into the demyelinating range in one and were absent in 14 . Table 2 shows the distal CMAP and TD values in those segments which had CB in our IvIg responsive patients.

\section{Previous criteria for CB}

We compared our definition of $\mathrm{CB}$ with some of the other definitions available in the literature ${ }^{12} 14171921$ given that some of the latter criteria may be too stringent for day-to-day diagnosis of MMN. When comparing, we chose the widest and therefore the most inclusive criteria from these papers, when available. This meant that criteria used for "possible

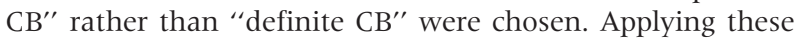
criteria to the nerves used in this study, no CB was detected in $20 \%^{12}{ }^{14}{ }^{17}$ to $30 \%{ }^{21}$ of IvIg responsive patients and was missed in up to $57 \%$ of the nerves (fig 1 ).

Proximal segments accounted for $36 \%(4.8) \%$ of the CBs, with relatively narrow variation between results using different criteria (figs 2 and 3).

\section{DISCUSSION}

This study sought a practical electrophysiological definition of $\mathrm{CB}$ in patients with MMN who might respond to IvIg therapy. All our patients fulfilled the clinical criteria for MMN and responded to IvIg. Early weakness of finger or wrist extension and a relative lack of wasting in severely weakened muscles, seen in some of our patients, are typical of this disorder. ${ }^{4}$ Three patients had worsened with steroid treatment but subsequently responded to IvIg. ${ }^{8}$ Our clinical and electrophysiological diagnoses of MMN were supported by the sustained improvements that have been maintained at follow up for up to 7 years.

The most useful measure of $\mathrm{CB}$ is a proximal reduction in the CMAP area. We found reductions greater than 23\% distally and $29 \%$ proximally to be predictive of IvIg response. These values were defined from normal controls $($ mean+3 SD), but even if the highest percentage drop that still distinguishes IvIg responsive MMN patients was taken, the levels could be raised to $37 \%$ distally and $50 \%$ proximally 


\begin{tabular}{|c|c|c|c|c|c|}
\hline Patient number & Nerve & Segment & Distal CMAP amplitude (mV) & CB (\%) & TD (\%) \\
\hline \multirow[t]{3}{*}{1} & RU & D & 4.0 & 31 & 22 \\
\hline & & $\mathrm{P}$ & & 82 & $37^{*}$ \\
\hline & LU & D & 3.6 & 68 & $44^{*}$ \\
\hline \multirow[t]{3}{*}{2} & LM & D & 1.4 & 52 & -7 \\
\hline & LU & P & 4.8 & 49 & 27 \\
\hline & RU & $P$ & 5.5 & 40 & $123^{*}$ \\
\hline \multirow[t]{2}{*}{3} & LM & D & 1.7 & 40 & $53^{*}$ \\
\hline & $\mathrm{RU}$ & D & 2.8 & 39 & -15 \\
\hline \multirow[t]{3}{*}{4} & $\mathrm{RM}$ & D & 12.1 & 74 & 29 \\
\hline & LU & $\mathrm{P}$ & 5.8 & 78 & 15 \\
\hline & LM & D & 7.3 & 78 & $40^{*}$ \\
\hline \multirow[t]{6}{*}{5} & RM & D & 11 & 36 & 23 \\
\hline & & $\mathrm{P}$ & & 38 & 9 \\
\hline & LM & D & 5.5 & 53 & 5 \\
\hline & & $\mathrm{P}$ & & 31 & 4 \\
\hline & RU & D & 8.4 & 27 & 1 \\
\hline & LU & D & 13.4 & 32 & 13 \\
\hline \multirow[t]{6}{*}{6} & RM & D & 10.9 & 25 & 14 \\
\hline & & P & & 40 & 7 \\
\hline & LM & D & 5.2 & 52 & -15 \\
\hline & $\mathrm{RU}$ & D & 8.2 & 82 & $72^{*}$ \\
\hline & & $P$ & & 44 & -73 \\
\hline & LU & $P$ & 3.2 & 40 & $128^{*}$ \\
\hline \multirow[t]{7}{*}{7} & $\mathrm{RM}$ & D & 8.1 & 71 & -15 \\
\hline & & P & & 70 & $325^{*}$ \\
\hline & LM & D & 13.3 & 50 & $151^{*}$ \\
\hline & & $P$ & & 44 & 27 \\
\hline & RU & D & 4.1 & 51 & $76^{*}$ \\
\hline & & $P$ & & 36 & $46^{*}$ \\
\hline & LU & D & 6.1 & 46 & $131^{*}$ \\
\hline \multirow[t]{4}{*}{8} & $\mathrm{RM}$ & D & 13.8 & 81 & -8 \\
\hline & & P & & 45 & $97^{*}$ \\
\hline & RU & D & 6.7 & 32 & 3 \\
\hline & & P & & 53 & $86^{*}$ \\
\hline \multirow[t]{4}{*}{9} & RM & D & 14.4 & 29 & 20 \\
\hline & & $\mathrm{P}$ & & 43 & $105^{*}$ \\
\hline & LM & $P$ & 4.1 & 92 & $68^{*}$ \\
\hline & $\mathrm{RU}$ & $P$ & 6.5 & 46 & $56^{*}$ \\
\hline 10 & LU & D & 7.0 & 37 & $48^{*}$ \\
\hline
\end{tabular}

without altering sensitivity. We also found amplitude reductions greater than 33\% proximally or greater than $32 \%$ distally to be similarly useful.

Assessment of CB is necessary to differentiate MMN from other lower motor neurone syndromes. In the latter, pseudo$\mathrm{CB}$ may be caused by excessive interphase cancellation in the presence of small distal CMAP amplitudes and often polyphasic waveforms. ${ }^{14}$ In our study, we attempted to avoid this by excluding all nerves whose distal CMAP amplitudes were $<1.0 \mathrm{mV}$. TD due to non-uniform conduction is a feature of many chronic demyelinating neuropathies and in such cases interphase cancellation could result in the mistaken appearance of CB. ${ }^{14}$ This situation has led many authors to use TD either as an exclusionary or restrictive criterion for the diagnosis of $\mathrm{CB}_{1}^{1415171921}$ which although helpful for clinical research, leads to underdiagnosis of treatable patients. ${ }^{10}{ }^{17}$ Indeed, up to $30 \%$ of our patients would have remained electrophysiologically undiagnosed if such criteria had been applied. For instance, patient no. 16 described by Katz et $a l^{17}$ had a typical clinical picture of MMN and a raised anti-GMl antibody titre. However, her nerve conduction studies showed a 31\% drop in median CMAP area across the forearm, which did not constitute CB according to their criteria, but would have according to ours.

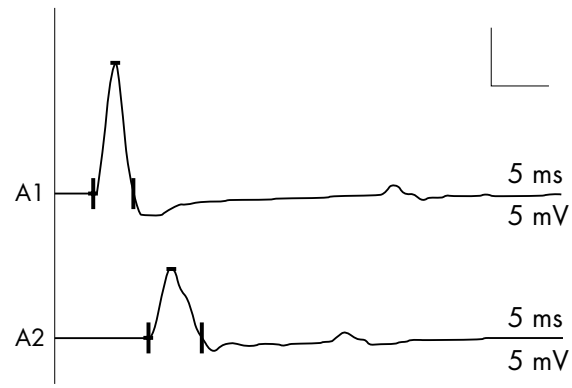

Before treatment

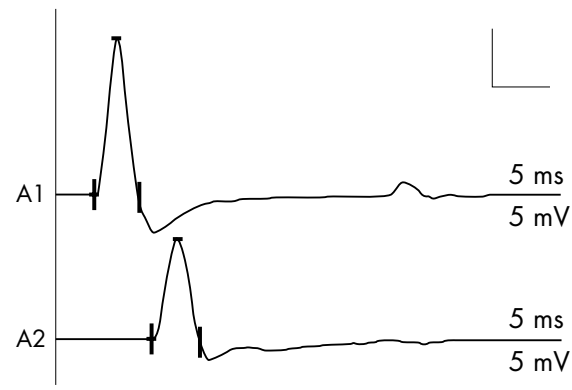

2 weeks after treatment

Figure 1 Measurement of degree of $\mathrm{CB}$ in the forearm median nerve by comparing abductor pollicis brevis $\mathrm{CMAP}$ amplitude following stimulation at the wrist (A1) and elbow (A2). Recordings are shown pre-treatment (left) and 2 weeks after $2 \mathrm{~g} / \mathrm{kg}$ body wt lvlg (right). 


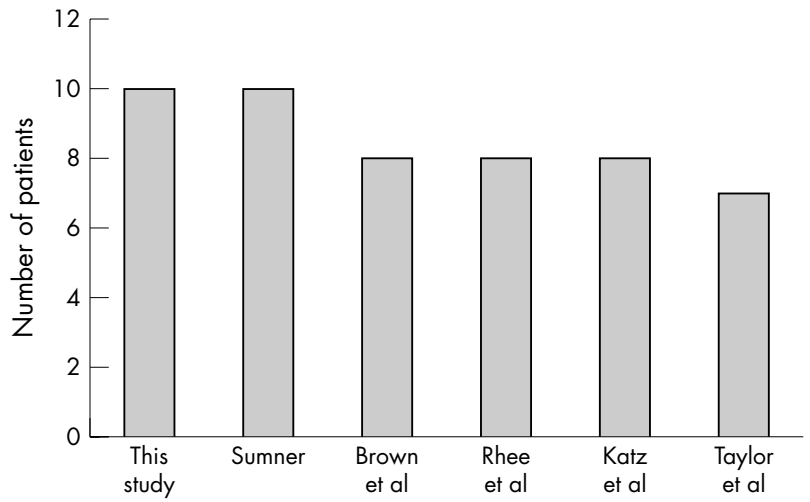

Figure 2 Number of patients who would show $C B$ in at least one nerve using different diagnostic criteria. Data are from Sumner, ${ }^{19}$ Brown and Feasby, ${ }^{12}$ Rhee et al, ${ }^{14}$ Katz et al, ${ }^{17}$ and Taylor et al. ${ }^{21}$

One could argue that more extensive neurophysiological assessment of all limbs could have identified more areas of $\mathrm{CB}$. This is not always practical in routine neurophysiological practice due to time, manpower, and financial constraints. The development of less stringent and more "useable" criteria is, therefore, of practical importance for predicting whether a lower motor neurone disorder will respond to IvIg. Pfeiffer et $a l^{24}$ suggest that "weak" criteria for CB interfere with prediction of response to IvIg. We argue that in the proper clinical context, where the suspicion of MMN is strong, a "weak" criterion is preferable in order to identify all responders, even at the cost of undertaking unrewarding treatment trials in a few non-responders. A recent study comparing IvIg responsive versus non-responsive subjects faced the dilemma of having to sacrifice some of sensitivity for more specificity and opted against it. ${ }^{10}$ In clinical practice, where the decision lies between diagnosing a treatable condition and an untreatable disorder with potentially grave prognosis, the issue of sensitivity gains priority even at the cost of losing some specificity. The need for less restrictive criteria has been highlighted in other ways. Cappellari et $a l^{18}$ suggested that the more marked drops in CMAP size may be preceded by smaller decrements which could nevertheless be suggestive of focal pathology in the appropriate clinical context.

TD may be as much an inherent part of MMN as is CB and the two frequently coincide in the same nerve segment. ${ }^{24} 25$ The relations between TD, drop in CMAP amplitude or area, conduction velocity, and DL are complex and may be dependent, among other factors, upon the relative balance between delay and block of axonal conduction among the faster and slower conducting fibres. ${ }^{26}{ }^{27}$ Preliminary studies suggest that TD improves following IvIg therapy in responsive patients, which improvement may be independent of measurable amplitude or area changes. ${ }^{27}$

It was of interest that the distribution of $\mathrm{CB}$ between proximal and distal segments in our patients remained remarkably similar even when different criteria from the literature were chosen. Proximal segments accounted for mean $36 \%$ of the CBs. This is also consistent with a recent study based on MR scans of the brachial plexus, ${ }^{10}$ which reported that 12 out of 31 (39\%) of the patients who fulfilled the clinical and electrophysiological criteria for MMN had abnormal T2-weighted images of the brachial plexus, sometimes associated with diffuse nerve swelling. In some patients proximal stimulation alone may reveal $\mathrm{CB}$, even when routine electrophysiological tests in the distal segments are normal. ${ }^{28}$ The importance of testing proximal nerve segments for CB needs particular emphasis.

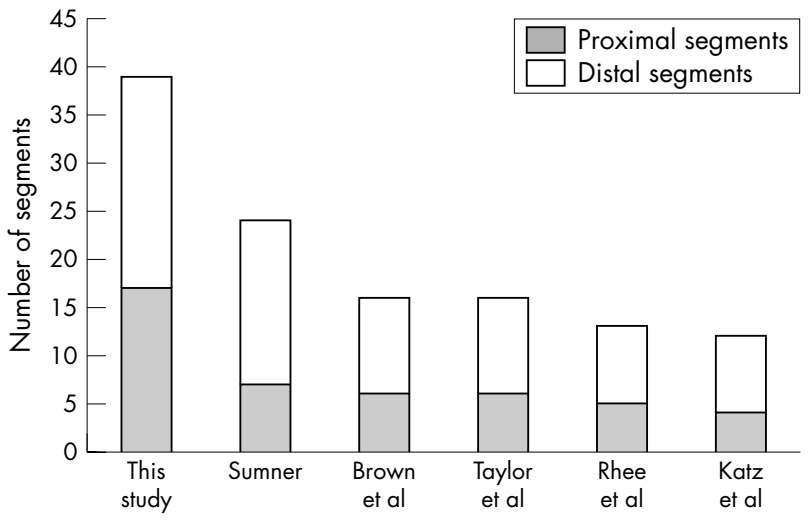

Figure 3 Number of proximal and distal segments which would show $\mathrm{CB}$ using different diagnostic criteria. Data are from Sumner, ${ }^{19}$ Brown and Feasby, ${ }^{12}$ Taylor et al, ${ }^{21}$ Rhee et al, ${ }^{14}$ and Katz et al. ${ }^{17}$

\section{Authors' affiliations}

A Ghosh, M Busby, M Donaghy, University Department of Clinical Neurology, Raddliffe Infirmary, Oxford OX2 6HE, UK

R Kennett, Department of Clinical Neurophysiology, Radcliffe Infirmary, Oxford OX2 6HE, UK

K Mills, Academic Neuroscience Centre, King's College Hospital, London SE5 9RS, UK

Competing interests: none declared

\section{REFERENCES}

1 Roth G, Rohr J, Magistris MR, et al. Motor neuropathy with proximal multifocal persistent conduction block, fasciculations and myokymia. Evolution to tetraplegia. Eur Neurol 1986;25:416-23.

2 Chad DA, Hammer K, Sargent J. Slow resolution of multifocal weakness and fasciculation: a reversible motor neuron syndrome. Neurology 1986;36:1260-3.

3 Parry GJ, Clarke S. Multifocal acquired demyelinating neuropathy masquerading as motor neuron disease. Muscle Nerve 1988;11:103-7.

4 Bouche P, Moulonguet A, Younes-Chennoufi AB, et al. Multifocal motor neuropathy with conduction block: a study of 24 patients. J Neurol Neurosurg Psychiatry 1995;59:38-44.

5 Kaji R, Shibasaki H, Kimura J. Multifocal demyelinating motor neuropathy: cranial nerve involvement and immunoglobulin therapy. Neurology 1992:42:506-9.

6 Nobile-Orazio E, Meucci N, Barbieri S, et al. High-dose intravenous immunoglobulin therapy in multifocal motor neuropathy. Neurology 1993:43:537-44.

7 Chaudhry V, Corse AM, Cornblath DR, et al. Multifocal motor neuropathy: response to human immune globulin. Ann Neurol 1993;33:237-42.

8 Donaghy M, Mills KR, Boniface SJ, et al. Pure motor demyelinating neuropathy: deterioration after steroid treatment and improvement with intravenous immunoglobulin. J Neurol Neurosurg Psychiatry 1994;57:778-83.

9 Van den Berg LH, Kerkhoff H, Oey PL, et al. Treatment of multifocal motor neuropathy with high dose intravenous immunoglobulins: a double blind, placebo controlled study. J Neurol Neurosurg Psychiatry 1995:59:248-52.

10 Van den Berg-Vos RM, Franssen H, Wokke JH, et al. Multifocal motor neuropathy: diagnostic criteria that predict the response to immunoglobulin treatment. Ann Neurol 2000;48:919-26.

11 Federico $\mathrm{P}$, Zochodne DW, Hahn AF, et al. Multifocal motor neuropathy improved by IVIg: randomized, double-blind, placebo-controlled study. Neurology 2000;55:1256-62.

12 Brown WF, Feasby TE. Conduction block and denervation in Guillain-Barre polyneuropathy. Brain 1984;107:219-39.

13 Cornblath DR, Sumner AJ, Daube J, et al. Conduction block in clinical practice. Muscle Nerve 1991;14:867-71.

14 Rhee EK, England JD, Sumner AJ. A computer simulation of conduction block: effects produced by actual block versus interphase cancellation. Ann Neurol 1990;28:146-56.

15 Chaudhry V, Corse AM, Cornblath DR, et al. Multifocal motor neuropathy: electrodiagnostic features. Muscle Nerve 1994;17:198-205.

16 Oh SJ, Kim DE, Kuruoglu HR. What is the best diagnostic index of conduction block and temporal dispersion? Muscle Nerve 1994;17:489-93.

17 Katz JS, Wolfe GI, Bryan WW, et al. Electrophysiologic findings in multifocal motor neuropathy. Neurology 1997;48:700-7.

18 Cappellari A, Nobile-Orazio E, Meucci N, et al. Criteria for early detection of conduction block in multifocal motor neuropathy (MMN): a study based on control populations and follow-up of MMN patients. J Neurol 1997;244:625-30. 
19 Sumner AJ. Consensus criteria for the diagnosis of partial conduction block and multifocal motor neuropathy. In: Kimura J, Kaji R, eds. Physiology of ALS and related diseases. Amsterdam: Elsevier, 1997:221-7.

20 Olney R. Consensus criteria for the diagnosis of partial conduction block Muscle Nerve 1999;22:S225-9.

21 Taylor BV, Wright RA, Harper CM, et al. Natural history of 46 patients with multifocal motor neuropathy with conduction block. Muscle Nerve 2000;23:900-8.

22 Mills KR, Murray NM. Electrical stimulation over the human vertebral column: which neural elements are excited? Electroencephalogr Clin Neurophysiol 1986;63:582-9

23 Cornblath D, Albers $P$, Feasby $T$. Research criteria for diagnosis of chronic inflammatory demyelinating polyneuropathy (CIDP). Report from an Ad Hoc
Subcommittee of the American Academy of Neurology AIDS Task Force. Neurology 1991;41:617-8.

24 Pfeiffer G, Wicklein EM, Wittig K. Sensitivity and specificity of different conduction block criteria. Clin Neurophysiol 2000;111:1388-94.

25 Lange DJ, Trojaborg W, Latov N, et al. Multifocal motor neuropathy with conduction block: is it a distinct clinical entity? Neurology 1992;42:497-505.

26 Ghosh A. Axonal multifocal neuropathy without conduction block or other features of demyelination. Neurology 2002;59:1666-7.

27 Ghosh A, Virgincar A, Kennett R, et al. The effect of treatment upon temporal dispersion in lvlg responsive multifocal motor neuropathy. J Neurol Neurosurg Psychiatry 2005;76:1269-72.

28 Jaspert A, Claus D, Grehl H, et al. Multifocal motor neuropathy: clinical and electrophysiological findings. J Neurol 1996;243:684-92.

\section{NEUROLOGICAL PICTURE}

\section{Delayed recovery of ulnar neuropathy due to elbow warming}

A 26 year old student presented clinically with a right ulnar sensory neuropathy, thought to have resulted from compression at the elbow during late night studying. There was no previous history, nor family history, of compression neuropathy; serum glucose and inflammatory markers were not elevated. Nerve conduction studies confirmed marked slowing across the elbow on the right, compared with the left side. Plain $\mathrm{x}$ ray of the right elbow revealed no bony abnormality.

Despite resting the arm, hand weakness progressed over the next 6 weeks, with evidence of hand clawing. At this time the student confessed that, in an attempt to stimulate recovery himself, he had fashioned an "elbow warmer" from an electric blanket (fig), which had been worn continuously over the previous weeks. He was instructed not to use it any more, and referred immediately for surgical intervention following which his weakness resolved.

Ulnar neuropathy at the elbow (UNE) remains a controversial entity, both in terms of neurophysiological diagnosis and subsequent management. ${ }^{1}$ UNE often improves with conservative management and simple elbow rest, but surgical referral is advised where there is progression of motor symptoms. Although it is not possible to be certain that the use of the "elbow warmer" worsened this patient's neuropathy, neurophysiology is influenced by temperature. ${ }^{2}$ Heat induced conduction block has been described in the setting of carpal tunnel syndrome, ${ }^{3}$ and cooling has been successfully used to aid recovery in common peroneal nerve compressive neuropathy. ${ }^{4}$ It is therefore conceivable that this home-made device prevented spontaneous recovery in this patient, necessitating surgical referral.

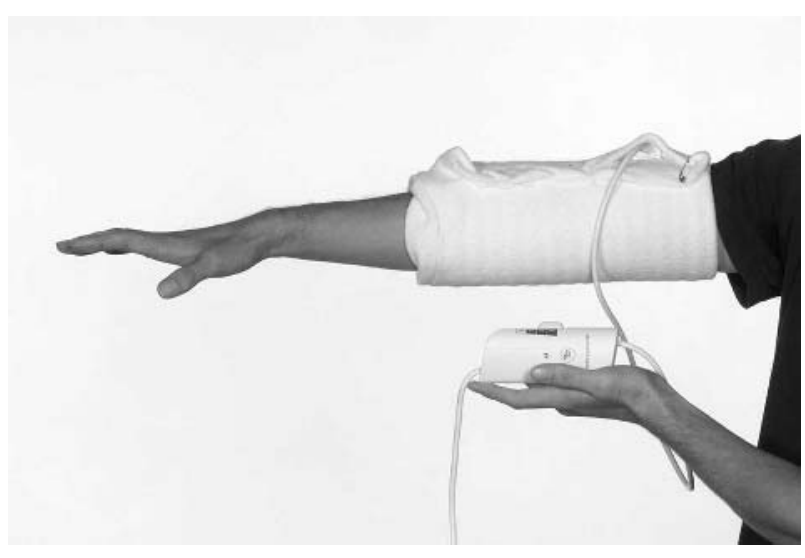

Correspondence to: Martin R Turner, Department of Neurology, The Radcliffe Infirmary, Woodstock Road, Oxford, OX2 6HE, UK; turnermr@doctors.org.uk

Patient consent has been obtained for publication of figure 1

Competing interests: none declared

\section{References}

1 Campbell WW. Ulnar neuropathy at the elbow. Muscle Nerve 2000;23:450-2.

2 Denys EH. AAEM minimonograph \#14: The influence of temperature in clinical neurophysiology. Muscle Nerve 1991;14:795-811.

3 Wang AK, Raynor EM, Blum AS, et al. Heat sensitivity of sensory fibers in carpal tunnel syndrome. Muscle Nerve 1999;22:37-42.

4 Rutkove SB. Focal cooling improves neuronal conduction in peroneal neuropathy at the fibular neck. Muscle Nerve 2001;24:1622-6. 